\title{
THEORY OF TWO-PHASE FLOW OF FLUID WITH RIGID ELLIPSOIDAL PARTICLES
}

\author{
NGUYEN VAN DIEP, NGUYEN HONG PHAN \\ Institute of Mechanics, NCNST of Vietnam
}

\begin{abstract}
SUMMARY. In the paper [1] the general continuum theory has been developed for twophase of fluid with deformable particles of arbitrary form, where the microdeformation of the particles and the relative motion between phases are taken into account. The extended theory of irrotational flow of fluid cansed by a moving deformable body has been used to obtain the general expressions for the generalized induced mass tensore.

The simplest case, when the particles have a spherical form during the micro-deformation, has been considered in the paper [2].

This paper is devoted to the theory of two-phase flow of fluid with rigid ellipsoidal particles. The obtained equation system can be used to determine the characteristic mean velocity, the particle rotation, the generalized diffusion fux of particles, the mass densities, the volume concentration of particles, the inertia tensor and generalized induced mass tensors of particle.
\end{abstract}

\section{DETERMINATION OF THE GENERALIZED INDUCED MASS TENSORS AND INERTIA TENSOR OF RIGID ELLIPSOIDAL PARTICLES}

We consider the motion of incompressible fluid with rigid ellipsoidal particles. In this case there is no particle micro-deformation and the particles can only rotate with rolation velocity $\bar{\omega}$ and translate.

Then it can be shown [i] that the generalized induced mass tensors can be determined by following expressions.

$$
\begin{aligned}
& \bar{M}=-\frac{1}{2} \rho_{2} \int_{d s_{1}}\left(\bar{n}^{u}+\bar{\Phi}^{u} \bar{n}\right) d s_{1}^{\prime} \\
& \bar{L}=-\rho_{2} \int_{d s_{1}}\left\{\left[\bar{n}^{\omega}-\bar{\Phi}^{u}(\bar{n} \times \bar{\xi})\right]+\left[\bar{\Phi}^{\omega} \bar{n}-(\bar{n} \times \bar{\xi}) \bar{\Phi}^{u}\right]\right\} d s_{1}^{\prime}, \\
& \bar{N}=\rho_{2} \int_{d \theta_{1}}\left[\bar{\Phi}^{\omega}(\bar{n} \times \bar{\xi})+(\bar{n} \times \bar{\xi}) \bar{\Phi}^{\omega}\right] d s_{1}^{\prime}
\end{aligned}
$$

The inertia tensor of particles has a form

$$
\bar{I}=\rho_{1} \int_{d v_{2}} \bar{\xi}_{1} \bar{\xi}_{1} d v_{1}^{\prime}
$$


It is easy to show that $\bar{M}, \bar{N}, \bar{I}$ are polar tensors of 2 nd order, $\bar{L}$ - axial tensor of 2 nd order.

In the expressions (1.1) and (1.2) $d s_{1}^{\prime}$ and $d v_{1}^{\prime}$ are the surface and volume elements of particle, $d s_{1}$ and $d v_{1}$ - surface and volume of particle $[1], \bar{n}-$ a unit vector normal to a paticle surface, $\rho_{1}$ and $\rho_{2}$ - mass densities of particles and fluid, the operator $(x)$ - vector product. The potential vectors $\bar{\Phi}_{u}$ and $\bar{\Phi}_{\omega}$ satisfy the Laplace equations inside the fluid micro-volume element and the following conditions on the particle surface

$$
\begin{aligned}
& (\bar{n} \cdot \bar{\nabla}) \bar{\Phi}^{u}=\bar{n} \\
& (\bar{n} \cdot \bar{\nabla}) \bar{\Phi}^{\omega}=-\bar{n} \times \bar{\xi}
\end{aligned}
$$

In other words, the potential of fluid motion caused by transtating and rotating motion of particle has a form

$$
\Phi=\left(\bar{u}_{1}-\bar{u}_{2}\right) \cdot \bar{\Phi}^{u}+\bar{\omega} \cdot \bar{\Phi}^{\omega}
$$

where $\vec{u}_{1}$ and $\bar{u}_{2}$ are translating velocity of particles and fluid.

In the coordinate system connected with the principal axes of ellipsoidal particle the particle surface is determined by an equation

$$
\frac{X^{2}}{a^{2}}+\frac{Y^{2}}{b^{2}}+\frac{Z^{2}}{c^{2}}=1
$$

where $a, b$ and $c$ are principal radii of ellipsoid.

It can be shown that in this coordinate system vectors $\bar{\Phi}^{u}$ and $\bar{\Phi}^{\omega}$ can be determined [3] and they have following components

$$
\begin{aligned}
\Phi_{z}^{u} & =-\frac{X A}{2-A_{0}} ; \quad \Phi_{x}^{\omega}=\frac{\left(b^{2}-c^{2}\right) Y Z(B-C)}{2\left(b^{2}-c^{2}\right)+\left(B_{0}-C_{0}\right)\left(b^{2}+c^{2}\right)} \\
\Phi_{y}^{u} & =-\frac{Y A}{2-B_{0}} ; \quad \Phi_{y}^{\omega}=\frac{\left(c^{2}-a^{2}\right) Z X(C-A)}{2\left(c^{2}-a^{2}\right)+\left(C_{0}-A_{0}\right)\left(c^{2}+a^{2}\right)} \\
\Phi_{Z}^{u} & =-\frac{Z C}{2-C_{0}} ; \quad \Phi_{Z}^{\omega}=\frac{\left(a^{2}-b^{2}\right) X Y(A-B)}{2\left(a^{2}-b^{2}\right)+\left(A_{0}-B_{0}\right)\left(a^{2}+b^{2}\right)} ;
\end{aligned}
$$

In (1.6) $A, B$ and $C$ have the form

$$
\begin{aligned}
& A=a b c \int_{\lambda}^{\infty} \frac{d \alpha}{\left(a^{2}+\alpha\right) \sqrt{\left(a^{2}+\alpha\right)\left(b^{2}+\alpha\right)\left(c^{2}+\alpha\right)}} \\
& B=a b c \int_{\lambda}^{\infty} \frac{d \alpha}{\left(b^{2}+\alpha\right) \sqrt{\left(a^{2}+\alpha\right)\left(b^{2}+\alpha\right)\left(c^{2}+\alpha\right)}} \\
& C=a b c \int_{\lambda}^{\infty} \frac{d \alpha}{\left(c^{2}+\alpha\right) \sqrt{\left(a^{2}+\alpha\right)\left(b^{2}+\alpha\right)\left(c^{2}+\alpha\right)}}
\end{aligned}
$$

In the integrals (1.7) value $\lambda$ is positive solutions of the equation

$$
\frac{X^{2}}{a^{2}+\lambda}+\frac{Y^{2}}{b^{2}+\lambda}+\frac{Z^{2}}{c^{2}+\lambda}=1
$$

with values $X, Y, Z$ satified equation (1.5)

The values $A_{0}, B_{0}, C_{0}$ are values of $A, B, C$ at $\lambda=0$.

Taking into account (1.6) - (1.8), from (1.1) it can be proved that in the coordinate system $X, Y, Z$ the generalized induced mass tensors have following components 


$$
\begin{aligned}
& M_{x x}=\frac{4}{3} \pi a b c \rho_{2} \frac{A_{0}}{2-A_{0}} ; \quad M_{x y}=M_{y x}=0 \\
& M_{y y}=\frac{4}{3} \pi a b c \rho_{2} \frac{B_{0}}{2-B_{0}} ; \quad M_{y z}=M_{z y}=0 \\
& M_{z z}=\frac{4}{3} \pi a b c \rho_{2} \frac{C_{0}}{2-C_{0}} ; \quad M_{x z}=M_{z x}=0 \\
& L_{x x}=L_{y y}=L_{z z}=L_{x y}=L_{y x}=L_{x z}=L_{z x}=L_{y z}=L_{z y}=0 \\
& N_{x x}=-\frac{4}{15} \pi a b c \rho_{2} \frac{\left(b^{2}-c^{2}\right)^{2}\left(B_{0}-C_{0}\right)}{2\left(b^{2}-c^{2}\right)+\left(B_{0}-C_{0}\right)\left(b^{2}+c^{2}\right)} ; \quad N_{x y}=N_{y x}=0 \\
& N_{y y}=-\frac{4}{15} \pi a b c \rho_{2} \frac{\left(a^{2}-b^{2}\right)^{2}\left(C_{0}-A_{0}\right)}{2\left(c^{2}-a^{2}\right)+\left(C_{0}-A_{0}\right)\left(c^{2}+a^{2}\right)} ; \quad N_{y z}=N_{z y}=0 \\
& N_{z z}=-\frac{4}{15} \pi a b c \rho_{2} \frac{\left(a^{2}-b^{2}\right)^{2}\left(A_{0}-B_{0}\right)}{2\left(a^{2}-b^{2}\right)+\left(A_{0}-B_{0}\right)\left(a^{2}+b^{2}\right)} ; \quad N_{z x}=N_{x z}=0
\end{aligned}
$$

It is obvious that the values $M_{x x}, \ldots, N_{z z}$ in (1.9) will be determined only when the integrals (1.7) can be calculated. In the paper the explicit expressions of the integrals (1.7) are obtained in following cases

a. Case $a=b>c$

$$
\begin{aligned}
& M_{x x}=M_{y y}=\rho_{2} V_{0} \frac{A_{1}}{-A_{1}+2 A_{3}} ; \quad M_{z z}=\rho_{2} V_{0} \frac{-A_{2}}{A_{2}+A_{3}} ; \\
& N_{x x}=N_{y y}=-\frac{1}{5} \rho_{2} V_{0} \frac{\left(a^{2}-c^{2}\right)\left(A_{1}+2 A_{2}\right)}{2 A_{3}+\frac{a^{2}+c^{2}}{a^{2}-c^{2}}\left(A_{1}+2 A_{2}\right)} ; \quad N_{z z}=0 .
\end{aligned}
$$

Where we used the symbols

$$
\begin{aligned}
& A_{1}=\arcsin e-e \sqrt{1-e^{2}} ; \quad A_{2}=\arcsin e-\frac{e}{\sqrt{1-e^{2}}} \\
& A_{3}=\frac{e^{3}}{\sqrt{1-e^{2}}} ; \quad e=\frac{\sqrt{a^{2}-c^{2}}}{a} ; \quad V_{0}=\frac{4}{3} \pi a^{2} c .
\end{aligned}
$$

b. Case $a=b<c$

$$
\begin{gathered}
M_{x x}=M_{y y}=\rho_{2} V_{0} \frac{B_{2}}{B_{3}-B_{1}} ; \quad M_{z z}=-\rho_{2} V_{0} \frac{B_{1}}{B_{1}+B_{3}} ; \\
N_{x x}=N_{y y}=-\frac{1}{5} \rho_{2} V_{0}\left(c^{2}-a^{2}\right) \frac{B_{1}+\frac{1}{2} B_{2}}{\frac{a^{2}+c^{2}}{c^{2}-a^{2}}\left(B_{1}+\frac{1}{2} B_{2}\right)-B_{3}} ; N_{z z}=0
\end{gathered}
$$

In (1.11) the symbols $B_{1}, B_{2}, B_{3}$ are

$$
B_{1}=\ln \left|\frac{1-e}{1+e}\right|+2 e ; \quad B_{2}=\ln \left|\frac{1-e}{1+e}\right|+\frac{2 e}{1-e^{2}} ; \quad B_{3}=\frac{2 e^{3}}{1-e^{2}}
$$

It is easy to determine the inertia tensor of rigid ellipsoidal particle in the coordinate system $X Y Z$. Its components have following forms

$$
\begin{aligned}
& I_{x x}=I_{y y}=\frac{4}{15} \pi a^{2} c p_{1}\left(a^{2}+c^{2}\right) \\
& I_{z z}=\frac{8}{15} \pi a^{4} c \rho_{1} ; I_{x y}=I_{y x}=I_{z x}=I_{x z}=I_{y z}=I_{z y}=0
\end{aligned}
$$


In conclusions it has to emphasize that the expressions (1.10) - (1.12) determine explicitly the components of generalized induced mass tensors and inertia moment tensor of ellipsoidal particle in the coordinate sytem connected with principal axes of particle.

\section{BASIC EQUATIONS SYSTEM}

Suppose that the considered fluid is incompressible $\left(\rho_{2}=\right.$ const $)$, particles are absolutely rigid $\left(\rho_{1}=\right.$ const $)$ and have a ellipsoidal form.

In this case it can be shown that the components of tensors $\bar{I}, \bar{M}, \bar{L}, \bar{N}$ in the fixed corrdinate system with satisfy the following change equations

$$
\begin{aligned}
& \frac{d^{(a)} \bar{I}}{d t}+\left(\frac{\bar{J}^{a}}{\rho_{1}^{*}} \cdot \bar{\nabla}\right) \bar{I}=\bar{\omega} \times \bar{I}-\bar{I} \times \bar{\omega} ; \\
& \frac{d^{(a)} \bar{M}}{d t}+\left(\frac{\bar{J}^{a}}{\rho_{1}^{*}} \cdot \bar{\nabla}\right) \bar{M}=\bar{\omega} \times \bar{M}-\bar{M} \times \bar{\omega} \\
& \frac{d^{(a)} \bar{L}}{d t}+\left(\frac{\bar{J}^{a}}{\rho_{1}^{*}} \cdot \bar{\nabla}\right) \bar{L}=\bar{\omega} \times \bar{L}-\bar{L} \times \bar{\omega} ; \\
& \frac{d^{(a)} \bar{N}}{d t}+\left(\frac{\bar{J}^{a}}{\rho_{1}^{*}} \cdot \bar{\nabla}\right) \bar{N}=\bar{\omega} \times \bar{N}-\bar{N} \times \bar{\omega}
\end{aligned}
$$

If at the initial moment of time the particle principal axes coincide with the fixed coordinate system, then the expressions (1.9) - (1.12) can be considered as the initial conditions for solwing the equations system (2.1). Here we can see that $\bar{L} \equiv 0$.

The general equations system describing the motion of fluid with rigid ellipsoidal particles has the following form.

The equation of mass conservation for the mixture

$$
\begin{aligned}
& \frac{d^{(a)} \rho}{d t}+\rho\left(\bar{\nabla} \cdot \bar{u}_{a}\right)=-\bar{\nabla}\left[\left(1-\frac{a_{1} \rho_{2}^{*}}{a_{2} \rho_{1}^{*}}\right) \bar{J}^{\alpha}\right] ; \\
& \rho=\rho_{1}^{*}+\rho_{2}^{*} ; \quad \rho_{1}^{*}=\varphi \rho_{1} ; \quad \rho_{2}^{*}=(1-\varphi) \rho_{2} ;
\end{aligned}
$$

The particles concentration charge equation

$$
\begin{aligned}
& \frac{d^{(a)} \varphi}{d t}+\varphi \bar{\nabla} \cdot \bar{u}_{a}=-\frac{1}{\rho_{1}} \bar{\nabla} \cdot \bar{J}^{a} \\
& \bar{u}_{a}=\sum_{k=1}^{2} a_{k} \bar{u}_{k} ; \quad \sum_{k=1}^{2} a_{k}=1 ; \quad \bar{J}^{a}=\rho_{1}^{*}\left(\bar{u}_{1}-\bar{u}_{a}\right) ; \\
& \frac{d^{(a)}}{d t}(\ldots)=\frac{\partial}{\partial t}(\ldots)+\left(\bar{u}_{a} \cdot \bar{\nabla}\right)(\ldots) ;
\end{aligned}
$$

The balance equation for the momentum of mixture

$$
\begin{aligned}
. \rho \frac{d^{(a)} \bar{U}_{a}}{d t} & =\rho \bar{f}-\bar{\nabla} p+\bar{\nabla} \tau_{0}-\bar{\nabla} \times \bar{\tau}_{1}^{a}+\bar{\nabla} \cdot \bar{\tau}_{2}^{a}- \\
& -\frac{D^{(a)}}{D t}\left(\frac{a_{2} \rho_{1}^{*}-a_{1} \rho_{2}^{*}}{a_{2} \rho_{1}^{*}} \bar{J}^{a}\right)-\bar{\nabla} \cdot\left[\frac{\bar{J}^{a} \bar{J}^{a}}{\rho_{1}^{*}}\left(1+\frac{a_{1}^{2} \rho_{2}^{*}}{a_{1}^{2} \rho_{1}^{*}}\right)\right] ; \\
\frac{D^{(a)}}{D t}(\ldots) & =\frac{d^{(a)}}{d t}(\ldots)+[(\ldots) \cdot \bar{\nabla}] \bar{u}_{a}+(\ldots)\left(\bar{\nabla} \cdot \bar{u}_{a}\right),
\end{aligned}
$$

The equation for determining the generalized diffusion fux of particles 


$$
\begin{aligned}
& \frac{a_{2}^{2} \rho_{1}^{*}+a_{1}^{2} \rho_{2}^{*}}{a_{2}^{2} \rho_{1}^{*}} \frac{D^{(a)} \bar{J}^{a}}{D t}+\frac{a_{2}^{3} \rho_{1}^{*}-a_{1}^{3} \rho_{2}^{*}}{a_{2}^{3} \rho_{1}^{*}} \bar{\nabla} \cdot\left(\frac{\bar{J}^{a} \bar{J}^{a}}{\rho_{1}^{*}}\right)+\frac{a_{1}}{a_{2}} \bar{J}^{a} \frac{d^{(a)}}{d t}\left(\frac{a_{1} \rho_{2}^{*}}{a_{2} \rho_{1}^{*}}\right)- \\
& -\frac{a_{1}}{a_{2}} \frac{\bar{J}^{a}}{\rho_{1}^{*}}\left(\bar{J}^{a} \cdot \bar{\nabla}\right)\left(\frac{a_{1}^{2} \rho_{2}^{*}}{a_{2}^{2} \rho_{1}^{*}}\right)+n\left(1+\frac{a_{1}}{a_{2}}\right) \bar{M} \cdot\left\{\frac{d^{(a)}}{d t}\left[\left(1+\frac{a_{1}}{a_{2}}\right) \frac{\bar{J}^{a}}{\rho_{1}^{*}}\right]+\right. \\
& \left.+\left(\frac{\bar{J}^{a}}{\rho_{1}^{*}} \cdot \bar{\nabla}\right)\left[\left(1+\frac{a_{1}}{a_{2}}\right) \frac{\bar{J}}{\rho_{1}^{*}}\right]\right\}+\frac{M}{2}\left[\frac{d^{(a)} \bar{M}}{d t}+\left(\frac{\bar{J}^{a}}{\rho_{1}^{*}} \cdot \bar{\nabla}\right) \bar{M}\right] \cdot \bar{J}^{a}= \\
& =\rho_{1}^{*}\left(\bar{f}_{1}-\frac{a_{1} \rho_{2}^{*}}{a_{2} \rho_{1}^{*}} \bar{f}_{2}\right)-\rho_{1}^{*}\left(1-\frac{a_{1} \rho_{2}^{*}}{a_{2} \rho_{1}^{*}}\right) \frac{d^{(a)} \bar{u}_{a}}{d t}-\varphi\left[1-\frac{a_{1}(1-\varphi)}{a_{2} \varphi}\right] \bar{\nabla} p- \\
& -\rho_{1}^{*}\left(1+\frac{a_{1}}{a_{2}}\right)(\bar{\nabla} \mu)_{p, T}+\bar{\nabla} R_{0}^{a}-\bar{\nabla} \times \bar{R}_{1}^{a}+\bar{\nabla} \cdot \bar{R}_{2}^{a} ;
\end{aligned}
$$

The equation for determining the particles rotation

$$
\rho_{1}^{*} \bar{\sigma}=\rho_{1}^{*} \bar{\ell}+\bar{\tau}_{1}^{a}+\bar{\nabla} \lambda_{0}-\bar{\nabla} \times \bar{\lambda}_{1}+\bar{\nabla} \cdot \bar{\lambda}_{2}
$$

where

$$
\begin{aligned}
\rho_{1}^{*} \bar{\sigma}= & n(\bar{N}+\bar{I}) \cdot\left[\frac{d^{(a)} \bar{\omega}}{d t}+\left(\frac{\bar{J}_{1}^{a}}{\rho_{1}^{*}} \cdot \bar{\nabla}\right) \bar{\omega}\right]+ \\
& +\frac{n}{2}\left[\frac{d^{(a)}}{d t}(\bar{N}+\bar{I})+\left(\frac{\bar{J}_{1}^{a}}{\rho_{1}^{*}} \cdot \bar{\nabla}\right)(\bar{N}+\bar{I})\right] \cdot \bar{\omega}
\end{aligned}
$$

In the equations system (2.1) - (2.7) we use following symbols: $\rho$ - the mean mass density of the two-phase medium $\bar{u}_{a}$ - characteristic mean velocity, $\bar{J}^{a}$ - generalized duffusion flux of particles; $\bar{f}$ - density of the external body forces acting on an unit mass of mixture ; $p$ - thermodynamical pressure; $\bar{\tau}_{1}^{a}$ and $\bar{\tau}_{2}^{a}$ - antisymmetric and symmetric parts of stress tensor; $R_{0}^{a}, \bar{R}_{1}^{a}$ and $\bar{\Omega}_{2}^{a}$ trace, antisymmetric and symmetric parts of diffusion stress; $\bar{\ell}$ - density of the external body moments, $\lambda_{0}, \bar{\lambda}_{1}$ and $\bar{\lambda}_{2}$ - trace, antisymmetric and symmetric parts of moment stress tensor, $n$ - number of particles in an unit volume of the flow.

The obtained equation system (2.1) - (2.7) will be sufficient to determine all unknowns if the constitutive equations will be constructed. For this purpose one can use the results [1].

\section{CONCLUSION}

It has been constructed a full equations system, sufficient to determine all parameters of the flow of incompressible fluid with rigid ellipsoidal particles.

\section{ACKNOWLEDGEMENT}

This publication is completed with financial support from the National Basic Research Program in Natural Sciences. The authors with to express his gratitude for providing this excellent opportunity to carry out the research. 


\section{REFERENCES}

1. Nguyen Van Diep. Generalized diffusion theory of micromorphicly deformable particle-fluid continuum, Proceedings of the National Centre for Scientific Research of Vietnam, Vol 4, No 1. (1992).

2. Nguyen Van Diep. Generalized diffusion theory of micromosphicly deformable particle-fluid continuum and bubble-hiquid two-phase flows, J. of Mechanics (accepted for publication).

3. Kotrin N. E., Kibell I. A., Roze N. V. Theoretical Hydromechanics. Phyzmatgyz. Moscow. 1963.

\section{LÝ THUYÊTT DÒNG CHẢY HAI PHA CHẤT LÒNG MANG CÁC HAT CÚNG DANG ELLIPSOID}

Trong công trình này, chuyển động cùa chẩt long mang các hạt cứng dạng ellipsoid đã được khảo sát. Đã xác định được các ten xơ khối lự̣̂g nước kèm mở rộng về các phương trình biến

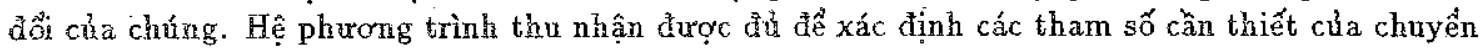
động của chất lỏng mang các hật cứng dạng ellipsoit.

\section{V竎 HộI NGHI KỸ THUẬT BIỄN VÀ DỊA CỰC QUỐC TẾ LÂN THỨ III}

(On the Third International Offshore and Polar Engineering Conference)

Hội nghị quốc tê về kỹ thuật biển và aịa cực lần thứ III (ICOPE-93) đã được tổ chác tại Singapor tù̀ ngày 06 đển 11 tháng 6 năm 1993. So vớ các lần trước (Lần thứ I: Edinburgh - 1991 và lần thú II: San Fransisco - 1992) Hội nghị lìn này đã tập hợp được rộng tãi hơn đội ngũ cán bộ

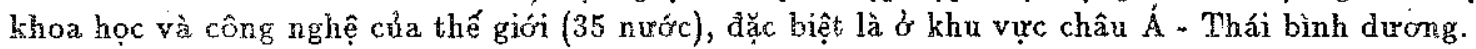

Gần 450 báo cáo khoa họ và công nghệ đ̛̉ược trình bày và trao đổi tại Hội nghị. Các báo cáo khoa học vầ công nghệ được công bố trong bộ tuyển tập gần 3000 trang bao gồn 04 tập với các nội dung nhur sau:

Vol. I - Global Offshore Energy Developments; Offshore Heavy Lift Operations; TLP, Fixed and Jack-kp Stryctures, Geotechnical Engineering, Reliability, Subsea System and Cost-efectiveness, Floating Production and Marginal Fields, Wave Energy and Power, Special Topics, Recources and Environment;

Vol. II - Computer Technology, ROV and Control, Risers, Mooring and Cables, Pipelines, International Polar Research, Ice, Structures and Scour;

Vol. III - Waves, Ocean Measurements, Hydrodynamic Forces, Vortex and Oscillations, Mo. tions and Nonlinear Dynamics, Higher - order Effects;

Vol. IV - Structural Mechanics and Analysis, Impact and Explosion, Materials, Welding, NDT and Fitress for Purpose, Fracture, Fatigue and Tubular Joints.

Hội nghị lần thứ IV sê được tổ chŕc tại Osaka (Nhật) từ ngày 10 đến 15 tháng 4 năm 1994 .

Có thể tham khá tài liệu cua các Hội nghị I - IIl và thể thức tham dự Hội nghị tại Viện Cơ học, 224 Đội Cẩ, Hà Nội. 